\title{
Automatic Assembly System for Modular Structure
}

\author{
Yuzuru Terada and Satoshi Murata \\ Tokyo Institute of Technology \\ string@mrt.dis.titech.ac.jp, murata@ dis.titech.ac.jp
}

\begin{abstract}
Automated construction is in high demand in many applications in the extreme environments such as space structure construction. In order to avoid exposure of human workers, construction machines are usually remote-controlled for such applications. Because of communication delay and narrow communication bandwidth, such systems require high-level expertise and also are ineffective and frustrating to the operators.

We have proposed a novel concept of automated construction called automatic Modular Assembly System. In this system, we use cubic modules to build structures, which have automatic connectors for handling by special assembler robots. The assembler robots are autonomous manipulators, which transport and assemble the modules. We have built a scaled prototype model and did some experiments of automatic assembly.

In this paper, we deal with an algorithm to construct large shell structures made of rectangular shaped panels which consist of many modules. We use a group of autonomous assembler robots for the construction and intelligent structure modules. We did some assembly simulations to evaluate the performance of autonomous-distributed algorithm.
\end{abstract}

Index Terms-modular robot; assembly; self-organize; connector.

\section{INTRODUCTION}

Automatic construction technique of buildings is more desirable than manual construction in extreme situations but that is not yet practical. For instance, all the large space structures must still be built by extra-vehicular activities (EVAs) of human astronauts. Therefore the astronauts are thereby exposed to dangers such as space debris and space radiation. Many other examples exist in civil engineering: huge offshore marine structures for ocean resources development, temporary structures before and after the strike of natural disasters [1], or those that allow restoration after disasters.

While remote controlled constructions are used in extreme scenes [2]-[4], there are many problems for its practical application. Construction works remote-controlled by human workers are inefficient due to communication delay and poor human interfaces. Large-scale construction has many processes where they use many types of robots for each process, making the management difficult and hindering the effective construction. Therefore we should automate these processes and contrive simpler process of construction.

We think the problem lies in the complexity of construction processes. Therefore we contrive to use powerful component of which buildings are made. Construction work should be simplified by using this kind of component. This can drastically reduce the total cost, which consists of robots, components and construction work

We have suggested the system, which contains such components as Structure Module and Automatic Assembly System for Modular Structure [5]. The purpose of this paper is to introduce the autonomous-distributed control algorithm for this system and simulations of automatic assembly.

\section{Automatic AsSEmbly SyStem FOR MOdUlaR STRUCTURE}

The modular structure has two features to simplify assembly process. First, every structure module is a regular hexahedron, which becomes a component of the modular structure. Second, it has 6 special designed connectors, which are genderless and rotation-symmetrical, in its walls.

Since the structure modules are regular hexahedron, we can practically make any structure with them. These modules can have built-in sensors, computers and so on.

The automatic assembler system for modular structure consists of structure modules and assembles robots. As shown in Fig.1, modular structure is based on cubic grid system defined by scale of structure module. The assembler robot connects on the modular structure, and its location and direction is indicated by discrete number on the grid. Because of this, robot's locomotion and assembly process is simplified and thus the operating efficiency is improved, reducing the total cost of the construction. 


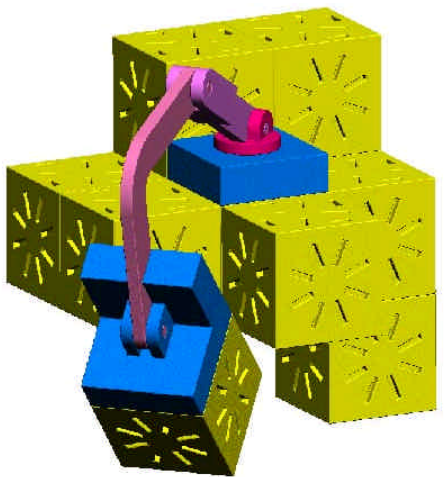

Fig.1 Modular structure and assembler robot

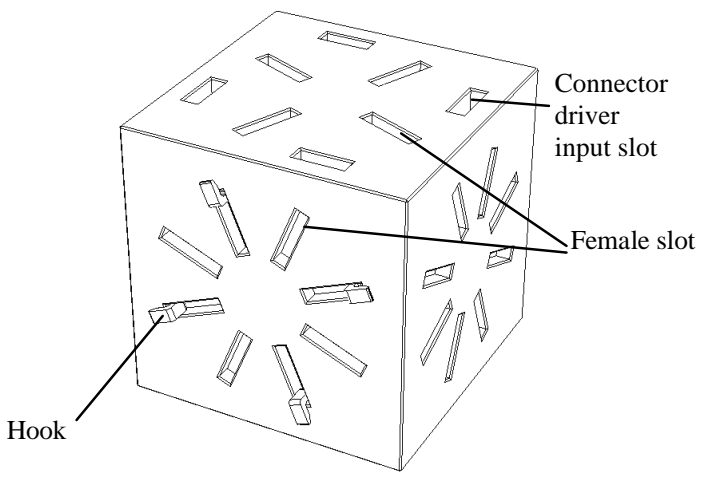

Fig.2 Structure module

Normally, locating an object in three-dimensional space requires six degrees of freedom; however in our system, four are sufficient because of its modularity. Each hand is equipped with male connectors that can connect to the module's female connectors.

The robot can move on the structure using an inchworm motion, repeating connection and disconnection actions (Fig.4). Connectors and links are sufficiently strong to hold and support the robot's entire body. Therefore, it can climb a vertical wall and hang onto a ceiling.

In this system, the assembler robot can construct almost all kinds of structure by these locomotion and assembly process.

We have built a scaled prototype model and did some experiments of automatic assembly (Fig.7).

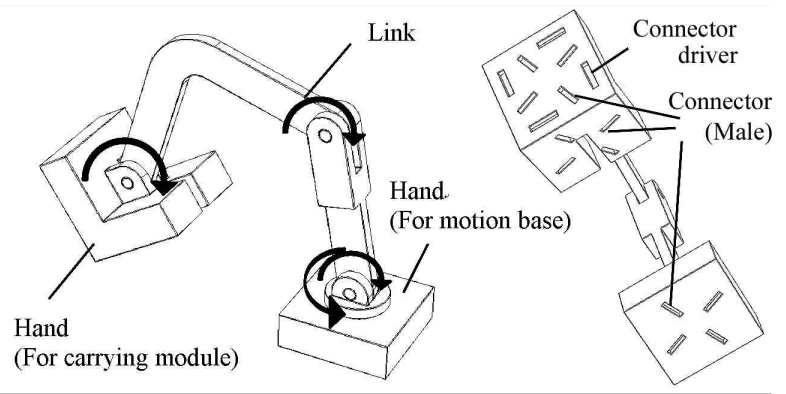

Fig. 3 Assembler robot

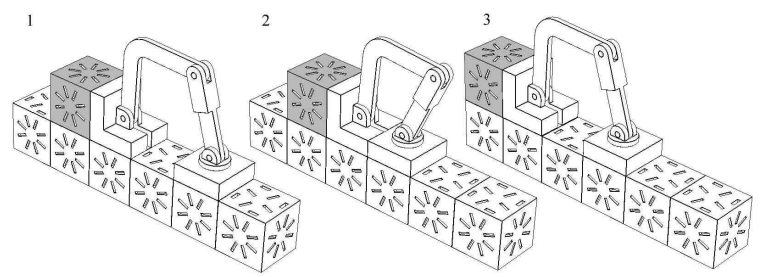

Fig. 4 Inchworm locomotion

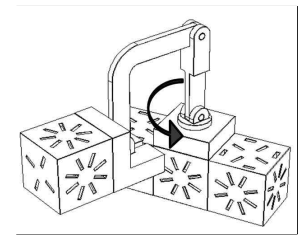

Fig. 5 Rotation

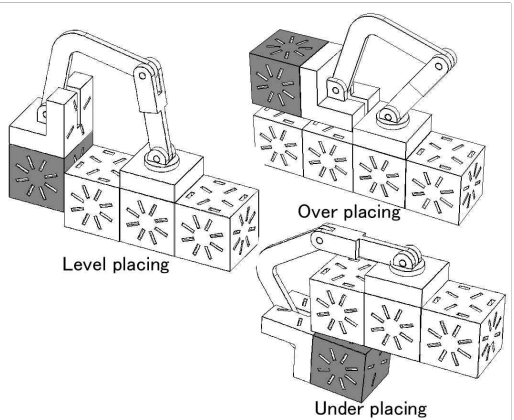

Fig.6 Assembling action

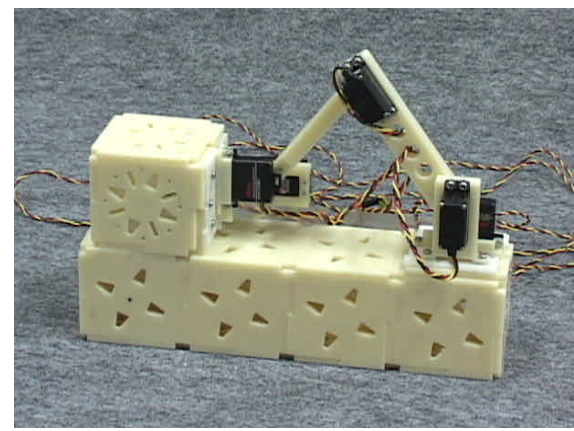

Fig.7 Experimental system

\section{ALGORITHM OF ASSEMBLY}

To assemble the large-scale modular structure efficiently, it is helpful to use large number of robots at a time. But planning the work for many robots becomes too complicated as the number of robots increases. This is more so in real world, for robots are affected by various disturbances from the environment and therefore making it difficult to synchronize them as planned. The best way is to give the robot autonomy and using autonomous-distributed control algorithm.

We propose a new algorithm which utilizes gradient field. The details are explained following sections. 


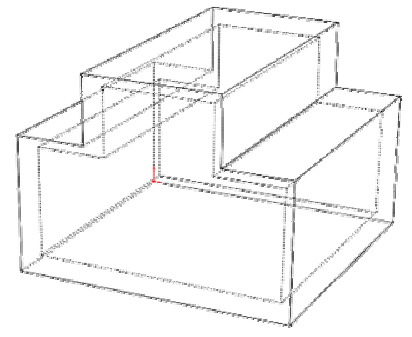

Fig. 8 Shell structure

\section{A. Assembly of shell structure}

In order to simplify the problem, let us think about assembling shell structure. Shell structure means the structure made of 1 layer board as shown at Fig.8.

Generally, shell structure made of square board can be unfolded to two-dimensional developments. Therefore, as a first goal, we made an algorithm to assemble planer structures of complicated shapes like developments of three-dimensional shell structures.

Fig.9 shows the simulation model. The robot assumed the same size as a structure module to simplify it. Here, the bottom line is module supply area and assembler robots can pick up structure modules. Robots go back and forth between growth front and module supply area to assemble the structure.

It is assumed that the structure modules contain microcomputer, contact sensor and local communication device. Target shape of structure is inputted to computers in advance. Modules can sense where they are by communicating with neighbors and which area should be filled. Growth front is formed by modules, next to which more modules area to be attached.

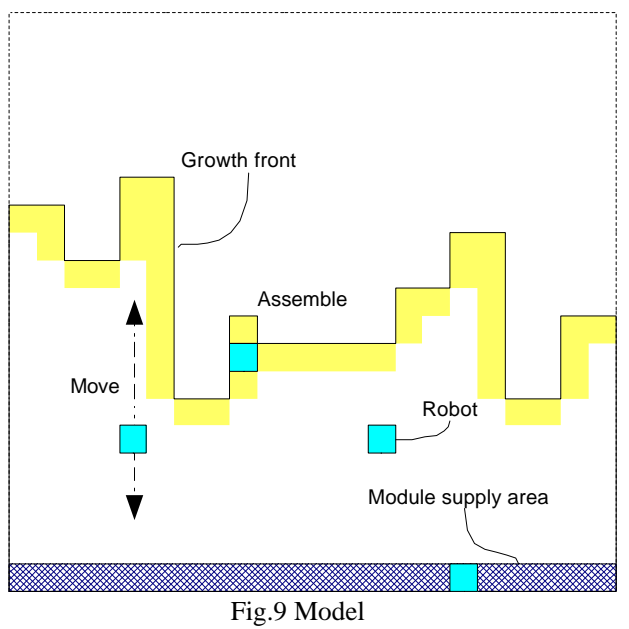

\section{B. Algorithm}

In order to indicate the location of growth front and module supply area to each robot, we use gradient field. The gradient field is implemented by only local communication among the structure modules, and it has high ability to transfer positional information to distant areas [6]. The gradient field is realized by distribution of potential values of structure modules. These values are high on the growth front and low in the module supply area. The robots can select their direction to go by sensing the gradient of the field.

Behavior of the robot is depends on whether the robot carries a module or not. With a module, a robot climbs the gradient field to go to the growth front. After placing the module to the assigned site, the robot goes down the field to go back to the module supply area, to get another module.

Because robots should know the location of growth front and module supply area from gradient field, it should be smooth and stable to indicate the destination.

The gradient field is realized by this diffusion equation:

$$
\Delta P=k \sum_{n=1}^{4}\left(P_{n}-P\right)
$$

Where $P_{\mathrm{n}}$ is neighbor's potential, $k$ is diffusion factor. This does not consider the leakage on the boundary of the shape. To stabilize the field, this equation is evaluated multiple times as a robot performs a single action.

The potential range is -1 to 1 . Growth fronts potential is:

$$
1-e^{-x / b}
$$

Where $\mathrm{x}$ is module number in the line, $\mathrm{b}$ is a constant. This equation prevents gathering too many robots around the short growth front. Module supply area's potential is -1 .

\section{Simulation}

Using this algorithm we do simulations which assemble a square and developed cube structure as shown on Fig.10.

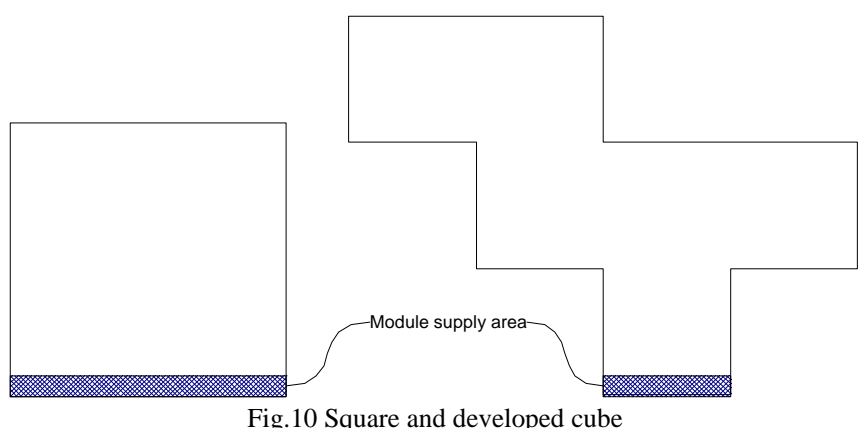

\section{A. Square assembly}

Fig. 11 is the result of the simulation to assemble square made of $28 \times 28=784$ modules. As the number of the robots increased, required time steps decreased and 'step $X$ robot' did not change significantly. The value of 'step $X$ robot' shows the total steps of robots working. It means that there are no inter-robot impediments in this simulation. Fig.13 shows the growth of the square with 10 robots. At first, they are concentrated in the center of the square. According to growth, the robots are deconcentrated and growth front becomes closer to a horizontal 
line. It seemed that robots are well controlled by the gradient field, which comes from the front of slow growth.

\section{B. Developed cube}

Fig. 12 is the result of the simulation to assemble a developed cube made of $10 \times 10 \times 6=600$ modules. As the number of robots increased, required time steps and 'step $x$ robots' decreased. Efficiency is the inverse proportion to the total steps, and there is a factor, which decreases the efficiency when the number of robots is small. Fig.14 shows the simulation of assembling a developed cube. In this figure, arrows indicate the direction of gradient. The arrow (in the white circle) points to the right and interrupts the flow to the upwards. Due to the arrow, some robots go to the right area, where the work has already been completed. This devious route is very narrow, and the number of trapped robot is almost same, if the total number of robot is changed. This seems to be the reason of low efficiency when using fewer robots. To assemble large and complicated structure, we should prevent this kind of loss.

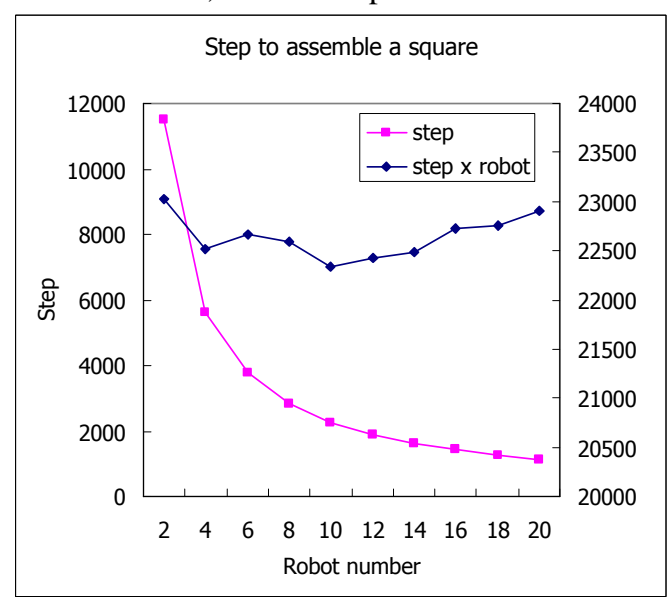

Fig.11 Result of square assembly

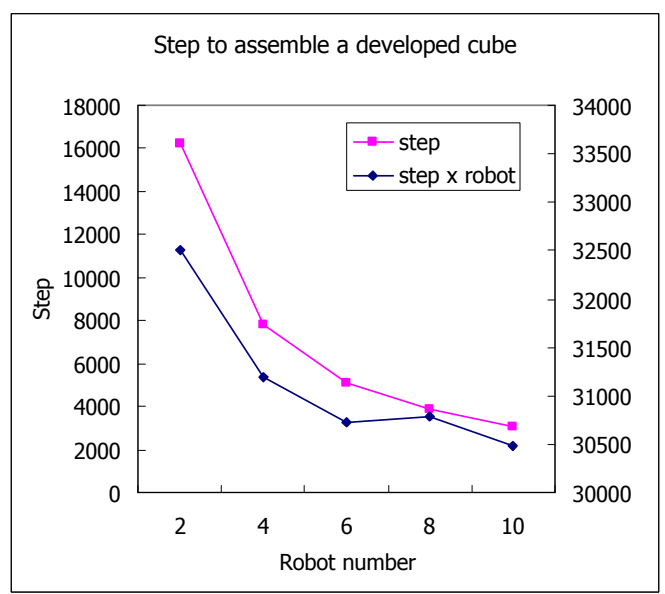

Fig.12 Result of developed cube assembly
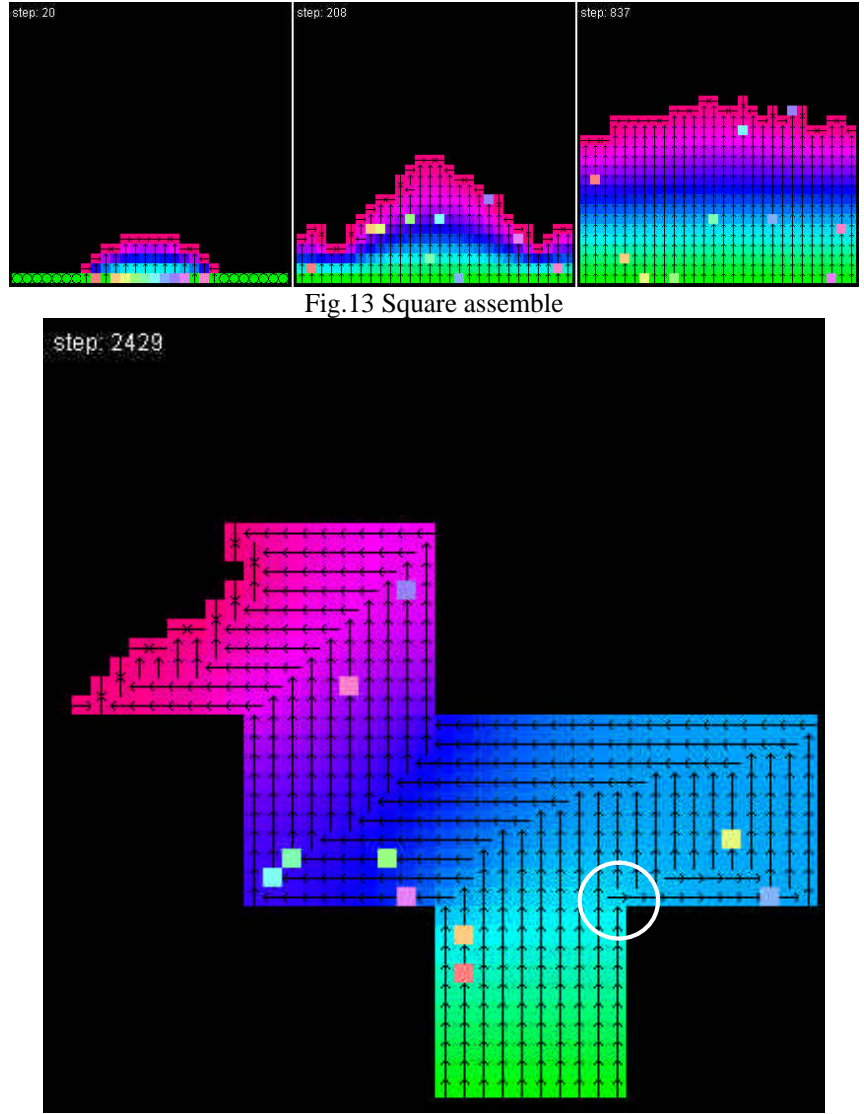

Fig.14 Developed cube assemble

\section{DISCUSSION}

We have shown our gradient-based algorithm can assemble two-dimensional structures. However, there are some problems yet to resolve. For example, in Fig.15, robots are congested at left end of these bridges because of the bridge is too narrow. One solution for this situation is to make bridges one-way. One is for outward and the other is for homeward. It can be made by self-organized process like heat convection. This technique seemed useful to construct a framed structure.

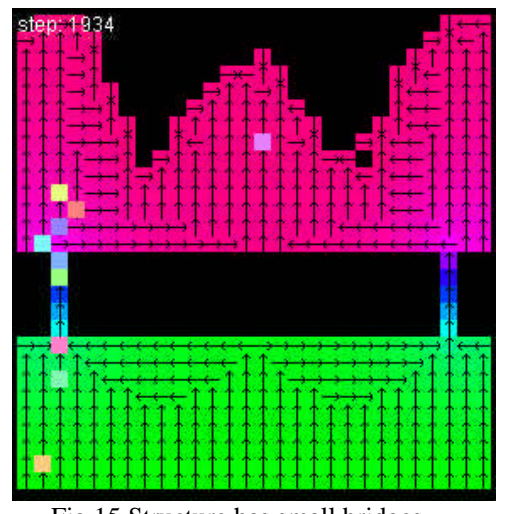

Fig.15 Structure has small bridges

In our algorithm, it is said that robot is controlled by structure with gradient. This characteristic can be used for interesting 
application. N. Inou suggested cell-automata based system, in which the structure optimizes itself to the stress [7]. This idea can be implemented in our system by using gradient for alert the too much stress. In addition, the structure can build intermediate structures to support construction. Fig.16 shows an example of this idea that is to keep balance before the two parts of the bridge are connected in the middle. It is good solution for large scale construction.

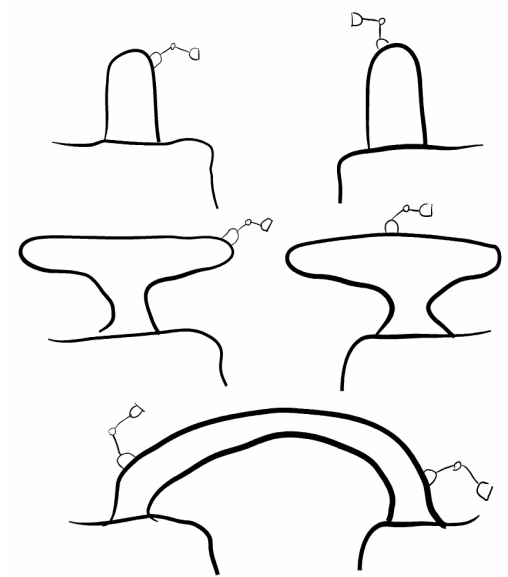

Fig.16 Assemble a bridge with intermediate structure

\section{CONCLUSION}

We have presented automatic assembly system for modular structure, and the advantages of remote-controlled assembly system. We showed an algorithm which uses gradient field and autonomous-distributed controlling assembler robots for modular structure. It is proved by simulation that the algorithm can assemble two-dimensional structures. We will improve this to apply to new experimental real model, and do substantive tests.

\section{REFERENCES}

[1] K. Chayama et al.: Implementation result of unmanned construction of a sabo dam using steel slit structure at Unzen Fugen-Dake, Proceedings of The 10th Symposium on Construction Robotics in Japan, pp.123-142, 2004

[2] William Whittaker et al.: Robotics for Assembly, Inspection, and Maintenance of Space Macrofacilities, AIAA-2000-5288

[3] Junichi Mitsunaga: Construction Machinery/Robot Business, Journal of Robotics Society of Japan, Vol. 20, No.7, pp.696-700, 2002

[4] Yuichi Ikeda, Takashi Shiokawa, Hironobu Kawakami, and Hidenori Miyajima: Development of an Automated Building Construction System (Part 3) - Improvement of System and Application of Super High-rise Hotel - the Obayashi Corp. Technical Lab. Report, No. 66, 2003

[5] Yuzuru Terada and Satoshi Murata: Automatic Assembly System for a Large-Scale Modular Structure -Hardware design of module and assembler robot- Proceedings of 2004 IEEE/RSJ International Conference on Intelligent Robots and Systems (IROS2004), pp.2349-2355, 2004

[6] K. Stoy: Controlling Self-Reconfiguration using Cellular Automata and Gradients, Proceedings of the 8th International Conference on Intelligent Autonomous Systems (IAS-8), pp. 693-702, 2004

[7] N. Inou et al.: Group Robots Forming a Mechanical Structure Development of Slide Motion Mechanism and Estimation of Energy Consumption of the Structural Formation -, Proceedings of IEEE International Symposium on Computational Intelligence in Robotics and Automation, pp.874-879, 2003 